\title{
Zn-doped $\mathrm{BaTiO}_{3}$ Materials: A DFT Investigation for Optoelectronic and Ferroelectric Properties Improvement
}

\author{
Luis H. S. Lacerda, Renan A. P. Ribeiro, Ageo M. de Andrade \& Sérgio R. de Lazaro
}

\section{Introduction}

Theoretical methods based on quantum mechanical simulations are an important tool to study material properties. Such methodologies are used for analyzing the electronic, optical, structural, pyro-, piezo- and ferroelectric properties of any materials. In case of crystalline semiconductors materials, these properties are highly dependent on chemical composition due to chemical bond character. Among the crystalline materials, the semiconductors materials stand out once are largely employed to develop electronic, optical devices, piezoelectric and memory devices because of its spontaneous polarization ${ }^{1-12}$. Then, the perovskite materials are an important group of materials since are the most abundant minerals in Earth. ${ }^{13}$ This material has $\mathrm{ABX}_{3}$ stoichiometry since $\mathrm{A}$ is a mono or bivalent cation; $\mathrm{B}$ is tetra or pentavalent cation and $\mathrm{X}$ is a non-metallic element, in most cases oxygen. As well as the other semiconductor materials, the properties of perovskite materials are dependent of chemical bonds and symmetry of crystalline structure.

Among the perovskite materials, a most important is $\mathrm{BaTiO}_{3}$ material (BTO). This material is a solid that can be found in five crystalline structures which differ according to central atom (Ti) in unit cell. The stability of each crystalline structure of BTO is dependent of temperature variation as showed in
Figure 1. At room temperature (298 K), the BTO has tetragonal structure. Such structure is characterized by Ti position out of symmetric center of unit cell resulting in properties, such as: optical, electronic, pyro-, piezo- and ferroelectric properties. ${ }^{14-16}$

In this work, the tetrahedral phase was investigated. This material has a band gap of 3.30 $\mathrm{eV}$ and is employed to development of integrated circuits, energy storage device, temperature coefficient resistance thermistor, piezoelectric sensors, sensing and monitoring devices, thin films, optoelectronic devices, actuators and others. ${ }^{16-21}$
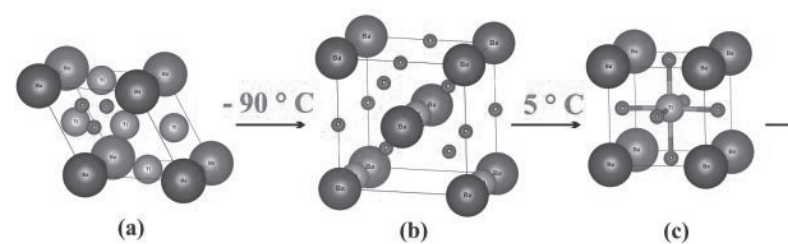

(c)

(b)

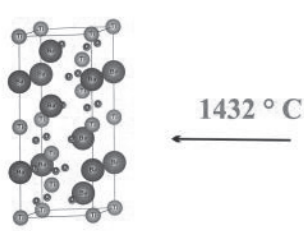

(e) $120^{\circ} \mathrm{C}$

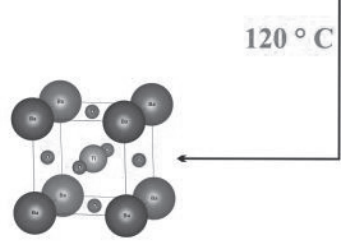

(d)
Figure 1. Crystalline Structures of barium titanate. a) Rhombohedra, b) Orthorhombic, c) Tetrahedral, d) Cubic and e) Hexagonal. 
Although BTO is a not a recent discovery, such material still is largely studied in order to improve innumerous devices, once its properties can be improved through of doping process. This process consists in adding a controlled amount of impurities in semiconductor structure that changes drastically the properties of a semiconductor material without changing its crystalline structure and controlling their properties. ${ }^{22,23}$ In case of $\mathrm{BTO}$, the doping process is possible by substitution of $\mathrm{Ba}$, Ti or $\mathrm{O}$ atoms ${ }^{.24-28}$ Among these substitutions, the replacement of $\mathrm{Ba}$ atoms for $\mathrm{Zn}$ atoms (BZTO) was not largely analyzed due to few works focused in this system. Such manuscripts evaluated only the structural, optical, electronic properties, the grain growth and photocatalysis application of Zn-doped BTO. ${ }^{24-26,29,30}$ Therefore, we propose a BZTO theoretical investigation based on DFT/ B3LYP to evaluate the effects of $\mathrm{Zn}$-doping in different amounts on BTO structural, electronic, optical and ferroelectric properties.

\section{Methods}

BTO material simulation was performed based on a tetragonal structure. This structure is composed by one $\mathrm{Ba}$ atom, one $\mathrm{Ti}$ atom and two $\mathrm{O}$ atoms arranged on $\mathrm{P} 4 \mathrm{~mm}$ (99) space group with lattice parameters $\mathrm{a}=$ $\mathrm{b}=3.98601 \AA, \mathrm{c}=4.0259 \AA$ and angles $\alpha=\beta=\gamma=$ $90^{\circ} .16$ The BZTO models was developed from unit cell expansion in the direction of axes a and $b$ resulting at 25 $100 \%$ doping (Table 1). For all models, the TZVP ${ }^{31}$ basis set was employed to describe the Ti, $\mathrm{O}$ and $\mathrm{Zn}$ atoms; whereas, the Ba atoms were described by Zagorac ${ }^{32}$ basis set employing the HAYWSC ${ }^{33}$ pseudopotential.

Table 1. Description of the unit cells used for simulating the $\mathrm{Zn}$-doping on $\mathrm{BaTiO} 3$ material.

\begin{tabular}{ccc}
\hline $\begin{array}{c}\text { Doped amount } \\
(\%)\end{array}$ & $\begin{array}{c}\text { Unit cell } \\
\text { expansion }\end{array}$ & $\begin{array}{c}\text { Ba atoms } \\
\text { replaced }\end{array}$ \\
\hline \hline $\mathbf{0}$ & $2 \times 2 \times 1$ & 0 \\
\hline $\mathbf{2 5}$ & $2 \times 2 \times 1$ & 1 \\
\hline $\mathbf{5 0}$ & $2 \times 2 \times 1$ & 2 \\
\hline $\mathbf{7 5}$ & $2 \times 2 \times 1$ & 3 \\
\hline $\mathbf{1 0 0}$ & $2 \times 2 \times 1$ & 4 \\
\hline
\end{tabular}

The calculation level applied was based on the Density Functional Theory (DFT) at set B3LYP hybrid functional ${ }^{34,35}$; SCF convergence was truncated in 10-8 Hartree and Mohnkhost-Pach ${ }^{36}$ method defined as $8 \times 8$ using CRYSTAL09 ${ }^{37,38}$ software. Vibrational calculations were also performed using optimized results for all models to evaluation of thermodynamic stability at room temperature $(298.5 \mathrm{~K})$ and $1 \mathrm{~atm}$. The theoretical results discussed in this manuscript are Density of States (DOS) Projections and Band Structure analysis. XCrysden ${ }^{39}$, ${ }^{40}$ Software was used for structural analysis. Personal computer using Ubuntu Linux operational system made up by quad-core AMD processor with $32 \mathrm{~GB}$ of RAM and data storage capability of $3.5 \mathrm{~TB}$ was used in all simulations.

\section{Results and Discussion}

\section{STRUCTURAL PROPERTIES}

The evaluation of $\mathrm{Zn}$ influence on structural properties of BTO material (Table 2) was performed through lattice parameters, unit cell angles, tetragonality factor (c/a) and $\mu$ structural coefficient (Equation 1). Such results indicate that unit cell symmetry was not affected by Zndoping. While Zn-doping amount increases, a, b and c lattice parameters decrease linearly (Figures 2). The linear variation of lattice parameters was evaluated based on Vegard's Law ${ }^{41,42}$ that provides a linear relationship between lattice parameters and impurity amount. According to this empirical rule the linear variation of lattice parameters with dopant amount variation indicates an ideal behavior for solid solution. Then, the $\mathrm{Zn}$ insertion in the BTO structure is characterized by an ideal behaviour indicating the possible formation of solid solution. The stability of BZTO materials are better discussed in Thermodynamic Stability Evaluation Section. For $50 \%$ model, the lattice parameter c are bigger than for BTO model due to the equal amount of $\mathrm{Ba}$ and $\mathrm{Zn}$ in crystalline structure which causes the perturbation through of replaced atoms distribution. However, the tetragonality index and $\mu$ values indicate that the tetragonal symmetry of unit cell is kept.

The lattice parameters are changed due to bond length decrease in crystalline structure, once for BTO model the average bond length for $\mathrm{Ba}-\mathrm{O}$ and $\mathrm{Ti}-\mathrm{O}$ are $2.865 \AA$ 
and $2.033 \AA$, respectively. As the Zn quantity increases, these values are reduced. Thus, for Zn-doped BTO at $100 \%$ such bonds have average bond length of $2.685 \AA$ and $1.928 \AA$, respectively. Although Ti - O bonds were changed, the $\mu$ coefficient structural evaluation indicates that the Ti position is slightly modified; therefore, it is expected the arising of ferroelectric and piezoelectric properties for BZTO materials are not smaller regarding to BTO.

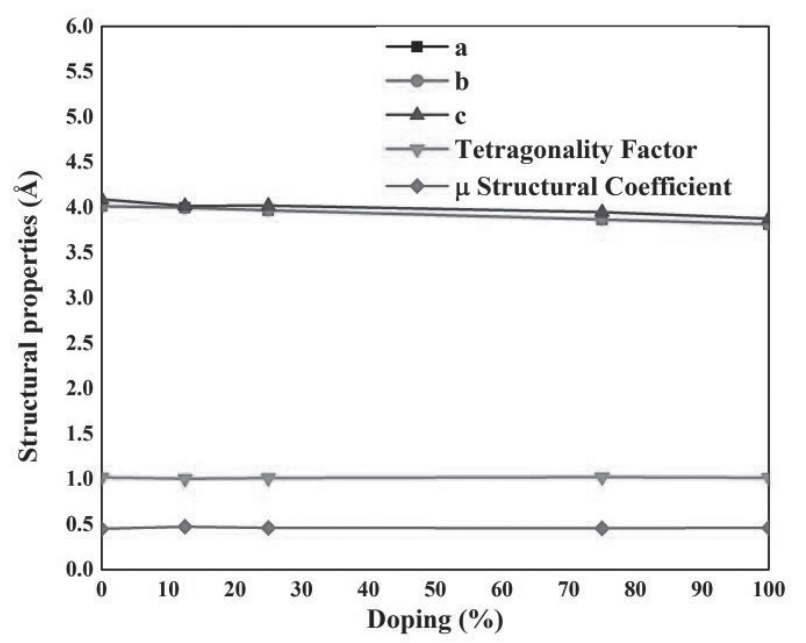

Figure 2. Variation of Lattice parameters (in $\AA$ ), unit cell angles (in degrees), Tetragonal factor $(\mathrm{a} / \mathrm{c})$ and structural coefficient $\mu$ for BTO and BZTO models according to dopant amount.

\section{ELECTRONIC PROPERTIES}

The electronic properties of BTO and BZTO materials were evaluated through the projected Density of States (DOS) for analysing models, evaluated from last five energy bands of the Valence Band (VB) and the first five energy bands of the Conduction Band (CB) featuring the band gap region $(\mathrm{Eg})$. For BTO model, the $\mathrm{O}$ atoms largely contribute to the composition on all $\mathrm{VB}$ and for lower energy levels of $\mathrm{CB}$ through $2 \mathrm{~s}$ and $2 \mathrm{p}$ orbitals; whereas, Ti atoms have low contribution for VB through of $3 \mathrm{~d}$ and $3 p$ orbitals and compose majority in $\mathrm{CB}$ through $3 \mathrm{~d}$ orbitals. In turn, Ba atoms lightly contribute for VB through 5sp and 6sp orbitals; while, its 3d orbitals contributed for $\mathrm{CB}$. The doping process has showed low influence on $\mathrm{O}$ and $\mathrm{Ba}$ contributions in $\mathrm{VB}$ and $\mathrm{CB}$. Nevertheless, the Ti contribution in $\mathrm{CB}$ was observed in a higher energy level than in BTO model. Zn contribution was made by $4 \mathrm{~s}$ orbitals that has low contribution in VB and average contribution in $\mathrm{CB}$. This insertion of $\mathrm{Zn}$ atoms shows a strong effect on the energy at the top of the VB in relation to the BTO material, causing degeneration of such energy levels, as well as reducing the contribution of the Ti atoms in CB.

\section{OPTICAL PROPERTIES}

The optical property of a solid is defined as the interaction between a solid and electromagnetic radiation. In case of semiconductor materials, the interaction only occurs with electromagnetic energy equal to or higher than $\mathrm{Eg}^{22,43}$. The optical property of BTO and BZTO material were evaluated through of $\mathrm{Eg}$ values (Table 3) showing that a decrease linearly with the $\mathrm{Zn}$ doping. Therefore, the presence of $\mathrm{Zn}$ atoms in the crystalline structure cause changes in the electronic structure from $\mathrm{Zn}$ orbitals insertion energy lower than Ti orbitals in the $\mathrm{CB}$. The band gap change after doping process is large, once for BTO the characteristic wavelength is located on Ultraviolet-Visible (UV-Vis); whereas, for BZTO at $100 \%$ the radiation characteristic is observed on Infrared region. As shown on Table 3, all the Eg are refered to an indirect excitation, except for $75 \%$ model that present a direct excitation along the $\mathrm{G}$ symmetry points.

Table 3. Theoretical results calculated for indirect and direct band gaps (Eg in $\mathrm{eV}$ ), wavelength (in $\mathrm{nm}$ ), electromagnetic radiation range in relation to doping amount (in \%).

\begin{tabular}{cccc}
\hline $\begin{array}{c}\text { Doping } \\
\mathbf{0}\end{array}$ & $\mathbf{E}_{\mathbf{g}}(\mathrm{eV})$ & $\boldsymbol{\lambda}(\mathbf{n m})$ & \multicolumn{1}{c}{ Radiation } \\
\hline \hline $\mathbf{0}$ & $\begin{array}{c}3.28 \\
(\mathrm{M}-\mathrm{G})\end{array}$ & 377.91 & $\begin{array}{c}\text { UV- } \\
\text { Visible }\end{array}$ \\
\hline $\mathbf{2 5}$ & $\begin{array}{c}2.89 \\
(\mathrm{R}-\mathrm{M})\end{array}$ & 429.33 & Visible \\
\hline $\mathbf{5 0}$ & $\begin{array}{c}2.21 \\
(\Lambda-\mathrm{G})\end{array}$ & 561.40 & Visible \\
\hline $\mathbf{7 5}$ & $\begin{array}{c}1.62 \\
(\mathrm{G}-\mathrm{G})\end{array}$ & 764.53 & Infrared \\
\hline $\mathbf{1 0 0}$ & $\begin{array}{c}0.75 \\
(\mathrm{~A}-\mathrm{G})\end{array}$ & 1656.50 & Infrared \\
\hline \hline
\end{tabular}


The Figure 3 shows the band-gap variation $\mathrm{g}$ ) for BZTO models in relation to BTO indicating a linear decrease. Then, all BZTO models have band-gap below to of BTO material. Such variation is caused by displacement of bottom of CB to a lower energy level than in BTO material due to $\mathrm{Zn}$ orbitals contribution. Figure 4 shows the results for top of VB and bottom of CB energy levels for all models investigated. These results indicate that the VB level for $\mathrm{Zn}$-doped models is slightly different of the energy level observed in BTO material. In order hand, the influence of Zn-doping process on $\mathrm{CB}$ energy level is strong, since the energy level is reduced largely.

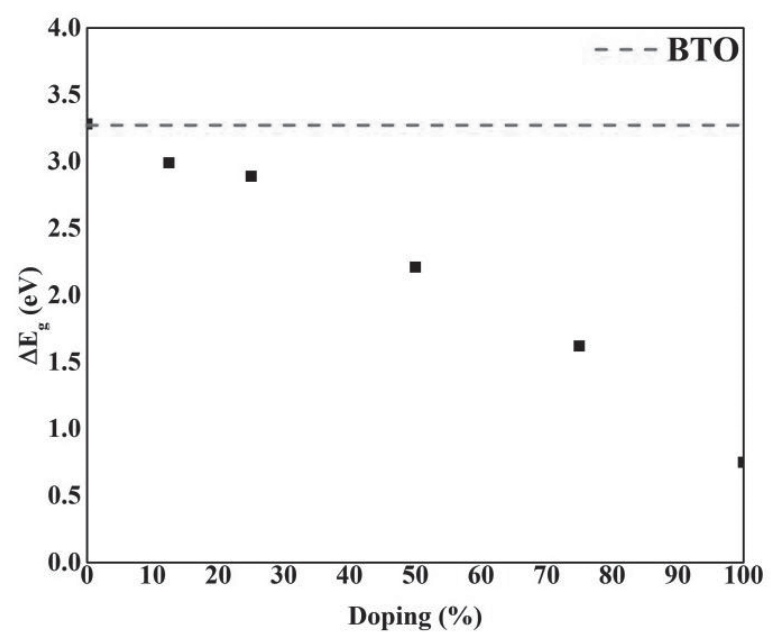

Figure 3. Theoretical results for BTO band gap according to Zn-dopant amount.

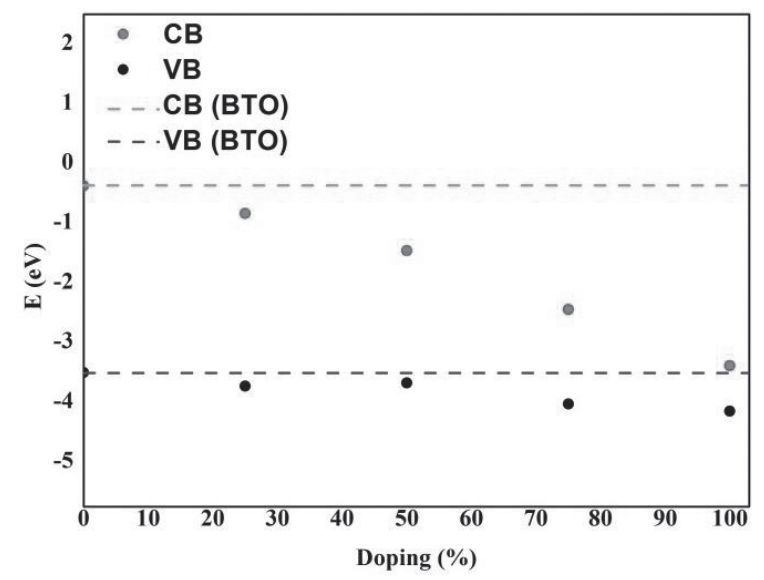

Figure 4. Energy levels calculated from DFT/B3LYP at the top of Valence Band (VB) and the bottom of Conduction Band (CB) according to $\mathrm{Zn}$-doping amount.

\section{FERROELECTRIC PROPERTIES}

Nowadays, the perovskite-type materials have been largely applied in the development of ferroelectric memories and charge storage devices because of high values for polarizability and dielectric constants presented by these materials. ${ }^{44-49}$ In a ferroelectric material the direction of charge polarization can be reverted applying an external electrical field, which is very important to the charge storage property.

In general, the dielectric constant $(\varepsilon)$ determines the charge storage capacity; whereas, the polarizability $(\alpha)$ describes how much a material is polarizable under an electrical field.43 These measures were developed by solid state theory, in order to better understand the ferroelectric property of the materials and they are dependent on the tensor matrix; which is directed similarly to the Cartesian coordinates system. For BTO and BZTO materials, the $\varepsilon$ and $\alpha$ amount were investigated based on components presented in Figure 5.

The $\varepsilon$ and $\alpha$ results for BTO and BZTO models are shown in the Table 4 and indicate that doping process improves largely the ferroelectric property because theoretically there is an increase in all tensor matrix components in relation to BTO; except for BZTO at $100 \%$ model, which shows a decrease for both $\varepsilon$ and $\alpha$ regarding to pure material. Thus, the higher values for $\varepsilon$ and $\alpha$ were observed to BZTO at $75 \%$ doping. The increase observed in relation to $\mathrm{Zn}$-doping, can be understood because the octahedron distortion in the crystalline structure that are responsible for the increase of the dielectric constants and polarizability. Another point is associated to the atoms random distribution in crystalline structure causing a lower symmetry in relation to BTO; consequently, the electronic density is randomky distributed providing ferroelectric and dielectric properties higher than those in the BTO.

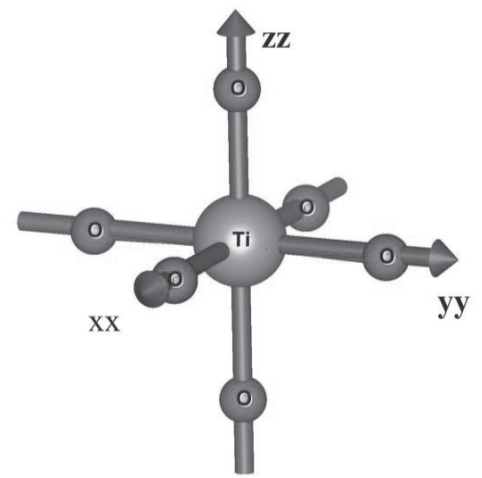

Figure 5. Octahedral sites for tetragonal-BTO and $\mathrm{xx}$, yy and $\mathrm{zz}$ components used for calculating dielectric constants and polarizability. 
Table 4. Polarizability $(\alpha)$ and dielectric constants $(\varepsilon)$ for BTO and BZTO models.

\begin{tabular}{ccccccc}
\hline \hline & \multicolumn{3}{c}{$\boldsymbol{\alpha}$} & \multicolumn{3}{c}{$\varepsilon$} \\
Dopagem (\%) & \multicolumn{3}{c}{ Componente } & \multicolumn{3}{c}{ Componente } \\
& $\mathbf{x x}$ & $\mathbf{y y}$ & $\mathbf{z z}$ & $\mathbf{x x}$ & $\mathbf{y y}$ & $\mathbf{z z}$ \\
\hline $\mathbf{0}$ & 154,1379 & - & 6216,2654 & 5,3564 & & 176,6886 \\
\hline $\mathbf{2 5}$ & 320,0193 & - & 8975,2729 & 5,7011 & & 66,9239 \\
\hline $\mathbf{5 0}$ & 295,1744 & 298,6174 & $301,6386 \cdot 10^{5}$ & 5,3724 & 5,4234 & $223,41 \cdot 10^{3}$ \\
\hline $\mathbf{7 5}$ & 333,3171 & 331,4823 & $474,9845 \cdot 10^{7}$ & 6,2531 & 6,2241 & $374,29 \cdot 10^{5}$ \\
\hline $\mathbf{1 0 0}$ & 162,264 & - & 150,181 & 6,359 & & 5,960 \\
\hline \hline
\end{tabular}

Table 5. Theoretical values for mixing Gibbs Free Energy variation $(\Delta \mathrm{Gmix})$ and Gibbs Free Energy variation in relation to pure BTO $(\Delta \Delta \mathrm{G})$ for BZTO materials

\begin{tabular}{|c|c|c|}
\hline Modelo (\%) & $\Delta \mathrm{sG}\left(\mathrm{kJ} . \mathrm{mol}^{-1}\right)$ & $\bar{c} \Delta \Delta \mathrm{G}\left(\mathrm{kJ} . \mathrm{mol}^{-1}\right)$ \\
\hline 0 & 0 & 0 \\
\hline 25 & -68.8969 & -51.4552 \\
\hline 50 & -41.6027 & -24.1609 \\
\hline 100 & 0 & -45.7471 \\
\hline
\end{tabular}

\section{THERMODYNAMIC STABILITY EVALUATION}

According to Vegard's law, the structural results for BZTO models indicate that this solid solution shows an ideal character; then, it is expected that these materials can be obtained by experimental techniques, once the crystalline structure is linearly changed from dopant amount. In literature, BZTO materials were synthetized from 0 to $10 \%$. In manuscript of Caballero and coauthors30 is not founded information of $\mathrm{Zn}$ solubility limit on BTO crystalline structure. Assuming that the Vegard's law prevision is not enough to determines the stability of BZTO materials, vibrational calculations were performed. Such vibrational calculations were based on DFT/B3LYP and we calculate the mixing Gibbs Free Energy ( $\Delta$ Gmix - Equation 2$)$ and Gibbs Free Energy in relation to BTO material ( $\Delta \Delta \mathrm{G}-$ Equation 3$)$.

$$
\begin{gathered}
(1-x) \mathrm{BaTiO}_{3}+{ }_{x} \mathrm{ZnO} \\
\rightarrow \mathrm{Ba}_{1-x} \mathrm{Zn}_{x} \mathrm{TiO}_{3} \\
\Delta \Delta G=\Delta G_{\text {mix }}-\Delta G_{\text {BTo }}
\end{gathered}
$$

Equation 2

Equation 3
The positive value for $\Delta \Delta \mathrm{G}$ indicates that these models are not favorable thermodynamically to be obtained the crystalline structure; while, the negative values suggest a thermodynamically factor favorable obtained the crystalline structure. The thermodynamically stability results for BTO and BZTO are present on Table 5. For all BZTO models, the DDGmix results are negatives in relation to BTO suggesting that such models are stable at room conditions. Then, these results corroborate with structural results provided by Vegard's law discussed before and they strongly suggest that BZTO materials a viable alternative to development of electronic, optical and ferroelectric devices and they are expected to show excellent fluorescence properties. ${ }^{50-52}$

\section{Conclusions}

We used DFT with periodic model to evaluate the structure, electronic, optical and ferroelectric properties for Zn-doping in barium tintanate. Electronic and optical properties showed that $\mathrm{Zn}$-doping are potential alternatives to be employed in electronic and optical devices, once the band-gap decrease changes the profile wavelength to the visible and infrared range of electromagnetic spectrum. The band-gap variation is caused by $\mathrm{Zn}$ atoms influencing as Valence Band as Conduction Band of $\mathrm{BaTiO}_{3}$ material. The ferroelectric property was evaluated for all models and presented that $\mathrm{Zn}$ atoms insertion is a good alternative to improve the ferroelectric properties of $\mathrm{BaTiO}_{3}$ materials. The vibrational frequencies and structure results for BZTO materials indicates that all models investigated are stable at room condition. 
Then, it is concluded tha the $\mathrm{Zn}$-doping on $\mathrm{BaTiO}_{3}$ crystalline structure improve electronic, optical and ferroelectric properties and it is a potential alternatives to application in ferroelectric devices employed in several electronic and optical devices, solar cells, and photocatalysis processes.

\section{Acknowledgments}

The authors acknowledge support from CNPq. CAPES and Fundação Araucária.

\section{References}

1. Z. J. Chew and L. Li, Materials Letters, 91, (2013).

2. C. Xun, L. Xiaomin, G. Xiangdong, L. Xinjun, Y. Chang, Y. Rui and J. Ping, Journal of Physics D: Applied Physics, 44, (2011).

3. M. K. Gupta, N. Sinha and B. Kumar, Journal of Applied Physics, 112, (2012).

4. F. Zhang, X. Li, X. Gao, L. Wu, F. Zhuge, Q. Wang, X. Liu, R. Yang and Y. He, Solid State Communications, 152, (2012).

5. S. Nedic, Y. Tea Chun, W.-K. Hong, D. Chu and M. Welland, Applied Physics Letters, 104, (2014).

6. M. K. Gupta and B. Kumar, Journal of Materials Chemistry, 21, (2011).

7. T. Qiuhong, W. Jinbin, Z. Xiangli, Z. Yichun, W. Qianjin, Z. Yi, $\mathrm{X}$. Zhang and S. Huang, Electron Devices, IEEE Transactions on, $58,(2011)$.

8. J. I. Sohn, S. S. Choi, S. M. Morris, J. S. Bendall, H. J. Coles, W.-K. Hong, G. Jo, T. Lee and M. E. Welland, Nano Letters, 10, (2010).

9. S. Lee, H. Kim, D.-J. Yun, S.-W. Rhee and K. Yong, Applied Physics Letters, 95, (2009).

10. D. U. Lee, E. K. Kim, W.-J. Cho, Y.-H. Kim and H. Im, Thin Solid Films, 521, (2012).

11. J.-W. Zhao, F.-J. Liu, H.-Q. Huang, Z.-F. Hu and X.-Q. Zhang, Chinese Physics B, 21, (2012).

12. Y. H. Kang, J.-H. Choi, T. I. Lee, W. Lee and J.-M. Myoung, Solid State Communications, 151, (2011).

13. M. W. Davidson and G. E. Lofgren, Journal of Geological Education, 39, (1991).

14. P. Erhart and K. Albe, Journal of Applied Physics, 102, (2007).

15. M. Yashima, T. Hoshina, D. Ishimura, S. Kobayashi, W. Nakamura, T. Tsurumi and S. Wada, Journal of Applied Physics, $98,(\mathbf{2 0 0 5})$.

16. R. A. Evarestov and A. V. Bandura, Journal of Computational Chemistry, 33, (2012).
17. P. Kumar, S. Singh, M. Spah, J. K. Juneja, C. Prakash and K. K. Raina, Journal of Alloys and Compounds, 489, (2010).

18. S.-H. Yao, J.-K. Yuan, P. Gonon, J. Bai, S. Pairis and A. Sylvestre, Journal of Applied Physics, 111, (2012).

19. Z.-X. Chen, Y. Chen and Y.-S. Jiang, The Journal of Physical Chemistry B, 105, (2001).

20. B. Wodecka-Dus and D. Czekaj, Archives of Metallurgy and Materials, 54, (2009).

21. J. A. Dawson, J. H. Harding, H. Chen and D. C. Sinclair, Journal of Applied Physics, 111, (2012).

22. H. L. Kwok, Electronic Materials (PWS Publishing Company, Boston, 1997).

23. A. R. West, Basic solid state chemistry (John Wiley \& Sons, Chichester, 2006), 2 ed. edn.

24. A. Y. Fasasi, M. Maaza, E. G. Rohwer, D. Knoessen, C. Theron, A. Leitch and U. Buttner, Thin Solid Films, 516, (2008).

25. A. Y. Fasasi, M. Maaza, C. Theron, P. Neethling, U. Buttner, A. Leitch and A. K. Chaudhary, Thin Solid Films, 516, (2008).

26. J.-P. Zou, L.-Z. Zhang, S.-L. Luo, L.-H. Leng, X.-B. Luo, M.-J. Zhang, Y. Luo and G.-C. Guo, International Journal of Hydrogen Energy, 37, (2012).

27. H.-S. Kwon, H.-I. Yoo, C.-H. Kim and K.-H. Hur, Journal of Applied Physics, 107, (2010).

28. C. Tian, Z. Yue, Y. Zhou and L. Li, Journal of Solid State Chemistry, 197, (2013).

29. A. C. Caballero, J. F. Fernández, C. Moure and P. Durán, Journal of the European Ceramic Society, 17, (1997).

30. A. C. Caballero, J. F. Fernández, C. Moure, P. Durán and Y.-M. Chiang, Journal of the American Ceramic Society, 81, (1998).

31. M. F. Peintinger, D. V. Oliveira and T. Bredow, J. Comput. Chem., 34, (2013).

32. D. Zagorac, K. Doll, J. C. Schön and M. Jansen, Chemistry - A European Journal, 18, (2012).

33. P. J. Hay and W. R. Wadt, J. Chem. Phys., 82, (1985).

34. A. D. Becke, J. Chem. Phys., 98, (1993).

35. A. D. Becke, Phys. Rev. A, 38, (1988).

36. C. Pisani, R. Dovesi and C. Roetti, Hartree-Fock ab initio Treatment of Crystalline Systems. (Springer Berlin Heidelberg, 1988), Lecture Notes in Chemistry, 48.

37. R. Dovesi, R. Orlando, B. Civalleri, C. Roetti, V. R. Saunders and C.-M. Zicovich-Wilson, Zeitschrift für Kristallographie, 220, (2005).

38. R. Dovesi, V. R. Saunders, C. Roetti, R. Orlando, C. M. ZicovichWilson, F. Pascale, B. Civalleri, K. Doll, N. M. Harrison, I. J. Bush, P. D'Arco and M. Llunell, CRYSTAL09 User's Manual (University of Torino, Torino, 2009). 


\section{Artigo Geral 69}

39. A. Kokalj, J. Mol. Graphics Modell., 17, (1999).

40. A. Kokalj, Comput. Mater. Sci., 28, (2003).

41. A. R. Denton and N. W. Ashcroft, Phys. Rev. A, 43, (1991).

42. L. Vegard, Zeitschrift für Physik, 5, (1921).

43. L. E. Smart, Solid state chemistry: an introduction. (Taylor \& Francis, Boca Raton, 2005), 3 ed. edn.

44. W. Wang, Q. Zhao, J. Xu and D. Yu, CrystEngComm, 14, (2012).

45. S. Cho, S. Kim, E. Oh, S.-H. Jung and K.-H. Lee, CrystEngComm, 11, (2009).

46. P. Chen, L. Gu and X. Cao, CrystEngComm, 12, (2010).

47. Y. Yang, J. Qi, W. Guo, Q. Liao and Y. Zhang, CrystEngComm, 12, (2010).

48. L. J. Brillson and Y. Lu, Journal of Applied Physics, 109, (2011).

49. Y. Lee, S. Lee, H. T. Ryu and D. Y. Kim, AIP Conference Proceedings, 1399, (2011).

50. J.-Z. Kong, A.-D. Li, H.-F. Zhai, H. Li, Q.-Y. Yan, J. Ma and D. Wu, Journal of Hazardous Materials, 171, (2009).

51. Z. Ali, S. Ali, I. Ahmad, I. Khan and H. A. Rahnamaye Aliabad, Physica B: Condensed Matter, 420, (2013).

52. Q.-J. Liu, N.-C. Zhang, F.-S. Liu, H.-Y. Wang and Z.-T. Liu, physica status solidi (b), 250, (2013).

\section{Luis H. da S. Lacerda*, Renan Augusto Pontes Ribeiro, Ageo M. de Andrade \& Sérgio R. de Lazaro}

Ponta Grossa State University, Department of Chemistry, PR

*E-mail:1hslacerda1@gmail.com 